\title{
Self-face perception: Individual differences and discrepancies associated with mental self-face representation, attractiveness and self-esteem
}

\author{
Fatima M. Felisberti ${ }^{1}$ and Kristina Musholt ${ }^{2}$ \\ 1. Kingston University, Kingston, United Kingdom \\ 2. University of Magdeburg, Magdeburg, Germany
}

\begin{abstract}
Self-face perception plays an important role in self-consciousness and personal identity as well as in social exchanges and well-being. Despite its significance, little is known about how individuals represent their faces internally. This study explored mechanisms of self-face perception in three experiments. First, participants chose from two images (self-face image vs. self-image with manipulated facial features) which one was their veridical image and which one they liked most. Afterwards, participants could (digitally) manipulate their facial features to increase their attractiveness (either to themselves or to an imagined other/s). Results showed that self-face recognition was better when veridical faces were paired with 'clones' with larger facial features or when all facial features were enlarged concurrently. Moreover, up to half of the participants preferred smaller noses and larger eyes and manipulated their self-images accordingly. State (but not trait) self-esteem was inversely correlated with eye, mouth and nose size manipulations made to increase one's attractiveness. The results indicate that a certain tolerance for error in self-face recognition might be required to maintain a consistent facial identity during one's lifespan. The discovered preference for neotenous features and discrepancies between one's perceived and one's veridical face and their link to state self-esteem are discussed. Keywords: self-face perception, attractiveness, state self-esteem, facial features, Self Discrepancy Theory.
\end{abstract}

Received 22 November 2013; received in revised form 03 February 2014; accepted 07 February 2014. Available online 27 June 2014.

\section{Introduction}

Face processing is typically fast and accurate and involves many well-documented cognitive processes (Bruce \& Young, 1986; Russel, Duchaine, \& Nakayama, 2009). The ability to recognise faces varies among individuals (Garrido, Duchaine, \& Nakayama, 2008), but despite the large quantity of research on face recognition, there are relatively few cognitive studies that address self-face processing.

Self-face perception differs from the perception of other faces and external body parts in that we can only view our faces indirectly (e.g., via photos or with the aid of mirrors). The images to which we have access are the same images that others can see. Children (15-to24-month-old) are already capable of a certain level of self-recognition in mirrors (Suddendorf, Simcock, \& Nielsen, 2007), an ability considered to be a pre-

Dr. Fatima M. Felisberti, Department of Psychology, Faculty of Social Sciences, Kingston University. Dr. Kristina Musholt, Department of Philosophy, University of Magdeburg. Correspondence regarding this article should be directed to: Fatima M. Felisberti, Dr. Fatima M. Felisberti, Department of Psychology, Faculty of Social Sciences, Penhryn road, London KT12EE, UK. E-mail: f.felisberti@kingston.ac.uk requisite for (reflective or conceptual) self-awareness (Gallup, 1970; Lewis, 2003) because it contributes to the construction and retrieval of a face representation that enables a subject to adopt the perspective of another onto themselves (Brédart \& Young, 2004; Gallup, 1998). Thus, self-face recognition is often hailed as a hallmark of (reflective) self-consciousness, which should be distinguished from other more basic forms of self-awareness (Zahavi \& Roepstorff, 2011).

Self-faces and unfamiliar faces involve separate cognitive processing as shown by studies involving visual adaptation (Rooney, Keyes, \& Brady, 2012) and gain mechanisms (Keyes \& Brady, 2010). Moreover, a unique cortical network for the processing of selfrelated body information, which partially overlaps with a cortical network for the extraction of body-related information (Devue et al., 2007; Hodzic, Muckli, Singer, \& Stirn, 2009), has been postulated. Studies also showed evidence that the cortical correlates of self-face recognition (i.e., right frontal cortex, parts of the insula) differ from and tend to be more lateralized than other types of face recognition (Devue \& Brédart, 2011; Kircher et al., 2001; Platek, Keenan, Gallup, \& Mohamed, 2004; Uddin, Kaplan, Molnar-Szakacs, Zaidel, \& Iacoboni, 2005). 
The recognition of one's face contributes to one's sense of identity and the study of the cognitive mechanisms that underlie self-face processing is essential to the understanding of a wide range of impairments, from minor cosmetic concerns to more serious disorders. More specifically, the mental representation of one's face that supports self-facerecognition is part of one's body image, which must be distinguished from the body schema. Whereas the latter is used for the (unconscious) guidance and control of actions, the former represents the (conscious) perceptual and conceptual representations of one's body and one's emotional attitude towards one's body (Gallagher, 1986). Thus, it is the body image that is distorted in disorders such as body dysmorphic disorder.

The body image - and (reflective) self-consciousness more generally - is closely linked to inter-subjectivity (Gallagher, 2012) and can be affected by the cultural and social context (Blais, Jack, Scheepers, Fiset, \& Caldara, 2008; Ma \& Han, 2009; Sugiura et al., 2012). For instance, according to Mead (1962), it is only by adopting the perspective of another person that we develop self-consciousness. According to this view, the self develops first in relation to a specific other (typically a child's father or mother) and then to a 'generalized other'. Building on this view, Higgins's Self-Discrepancy Theory (Higgins, 1987, 1989) distinguishes between the actual self and potential selves and between different standpoints on the self. With regard to the latter, Higgins argues that one can either reflect on oneself from one's own personal standpoint or from the standpoint of a significant other. With reference to the former, Higgins proposes three main domains of the self: the 'actual self' (one's representation of the attributes that someone-oneself or another-believes one actually possesses), the 'ideal self' (one's representation of the attributes that someone would ideally like one to possess), and the 'ought self' (one's representation of the attributes that someone believes one should, or 'ought to', possess). Importantly, discrepancies between the 'actual self' and one's 'selfguides' are associated with emotional discomfort and low self-esteem: the higher the discrepancy is, the lower the self-esteem (Higgins, 1987).

Self-esteem is typically defined by how a person feels about or values him- or herself. Heatherton and Polivy (1991) argue that self-esteem could be viewed either as a "state" or as a "trait"; fluctuations would occur around a stable baseline. Accordingly, their State Self-Esteem Scale (SSES, 20 items) is considered to be sensitive to environmental variability while addressing social, performance, and appearance factors. The SSES performance factor appraises the extent to which individuals feel their performance is valuable, whereas the appearance factor is considered to be the most sensitive to the manipulations that make one's physical appearance salient. The social factor refers to social anxiety and measures the extent to which individuals feel foolish, self-conscious, or uncomfortable about their public image.

Despite the significance of self-face recognition for self-consciousness and the sense of identity, little is known about how individuals represent their faces internally. This study aimed at addressing this gap in the literature by combining novel tasks designed to measure the accuracy of facial self-representation and the perception of self-face attractiveness with a measure of state self-esteem. More precisely, this study aimed:

i) To assess the participants' mental representation of the size of their facial features in relation to their real size (as seen in photographs).

ii) To examine if there are internal discrepancies between the perceived size of one's facial features and preferences for them (i.e., internal notions of self-face attractiveness) from the standpoint of oneself and from the standpoint of an imaginary other person(s).

iii) To investigate the link between such possible discrepancies and participants' state self-esteem.

Participants were first asked to choose from two images (self-face image vs. self-image with manipulated facial features) in order to indicate (a) which one was their veridical image and (b) which one they liked most. A follow-up experiment provided a more detailed assessment of the participants' ability to recognize individual facial features. In a third experiment, participants could (digitally) manipulate their facial features to increase their attractiveness (either to themselves or to an imagined other/s). In addition, participants were asked to complete the SSES questionnaire. Based on previous studies and on the Self-Discrepancy Theory, it was predicted that the higher the discrepancies in internal notions of selfface attractiveness are, the lower the self-esteem and the higher the magnitude of digital facial manipulation. Furthermore, based on the theory of sexual selection, it was predicted that participants would show a preference bias for neotenous (i.e., childlike) facial features, which are correlated with youth, health, and fertility (DeBruine, Jones, Tybur, Lieberman, \& Griskevicius, 2010; Kandrik \& DeBruine, 2012).

\section{Experiment 1}

The first experiment was designed to investigate the extent to which participants knew their facial features in the size domain. The experiment used a two-alternative forcedchoice paradigm (veridical $v s$. cloned images). Once the internal representation of one's facial features was known, it was possible to investigate self facial features preferences in relation to one's internal self-face image, which was carried out in the second part of this experiment.

\section{Method}

\section{Participants}

The participants $(N=33 ; 23$ females, Mean age $=$ $25, S D=7)$ were recruited using opportunity sampling 
and consisted of university students, staff, and their friends. The majority of the participants were recruited through the University SONA system for course credit and no cash payments were provided. The participants were informed that the experiment concerned self-face perception and that their face would be photographed. Participants provided written consent in accordance with the Faculty Research Ethics Committee and the British Psychological Society (BPS) ethics guidelines and performed in accordance with the ethical standards laid down in the 1964 Declaration of Helsinki. All of the participants had normal or corrected-to-normal vision.

\section{Stimuli}

Frontal digital photographs of the participants' full faces in a neutral expression were taken using a CANON EOS 450 camera. The background was light and uniform, and overhead lighting was used where possible to minimise shadows and decrease the need for posterior digital manipulation. The photographs were taken in communal areas on the university premises. Most participants showed no signs of wearing makeup, and if makeup was used, it was not conspicuous or clearly visible in the photographs. The photographs were taken at least 10 days prior to testing to leave time for test preparation and to weaken any detailed recollection of the photograph. Finer adjustments in luminance levels, contrast, red-eye filtering and the elimination of background shadows and distracters were performed using Photoshop CS5 (Adobe).

Distortions of facial features were created from individual digital images of faces ( $2080 \times 2560$ pixels) using a coarse mesh algorithm defined by four anchor points, which were fitted to the eyes, the nose, or the mouth areas (FaceFilter Studio 2, Reallusion). Size manipulation of the area of the eyes, the nose and the mouth was applied to each individual feature, or all three features were changed simultaneously (Figure 1). Each facial feature had the same amplitude in the $\mathrm{X} / \mathrm{Y}$ axis. Differences in area size between veridical self-faces and their clones were estimated using Photoshop CS5. The variation in measures of area size was typically in the range of $\pm 5 \%$. Eight clones of each veridical selfface feature were generated (i.e., four with larger facial features and four with smaller features).

\section{Procedure}

A recognition test was generated using E-Prime ${ }^{\circledR}$ 2.0 software (Psychology Software Tools Inc., Pittsburgh, PA). The test contained pairs of colour images: the actual self-face and one of its distorted clones. Each image in the test subtended approximately $11 \times 14$ degrees of visual angle at approximately $60 \mathrm{~cm}$ from the screen. The images (veridical and clone) were presented in pairs in the centre of the display monitor and separated from one another by a fixation point on a grey background (app. $1 \mathrm{deg}$ ). In each trial, the veridical self-face and its clones were alternated randomly between the left and right visual fields. In addition, the presentation of pairs for different facial features was interleaved and randomized.

In the first part of the experiment, the participants had to press ' 1 ' ' if the self-face was on the left side of the clone or ' 2 ' if the self-face was on the right side of the clone (i.e., left and right visual field, respectively). The pair of images remained on the screen until the participants chose the image that they thought was their veridical self-image. The participants were asked to make their judgements as rapidly and accurately as possible and when in doubt to base their judgements on 'first impressions'. The second part of the experiment resembled the first one except that instead of indicating which image was the veridical self-face, the participants had to choose which of the two self-images they liked most (veridical $v s$. clone). Here, ' 1 ' indicated they liked the image in the right and ' 2 ' indicated they liked the image in the left visual field.

\section{Data analysis}

A repeated measures ANOVA had as within-subject factors the facial features (4 levels: eyes, nose, mouth, EMN) and area sizes $(+25 \%$ and $-25 \%$, or + clone and -clone, respectively). EMN referred to simultaneous size changes to eyes, mouth and nose. In the first part of the experiment there were 8 trials repeated over 4 cycles (32 trials) and the same structure was repeated in the second part of the experiment, but with a different question. Greenhouse-Geisser adjustments to the degrees of freedom were performed when sphericity could not be assumed (Mauchly's sphericity test). Pairwise comparisons were performed with Bonferroni adjustments, and partial eta-square effect sizes were indicated by $p \eta^{2}$.

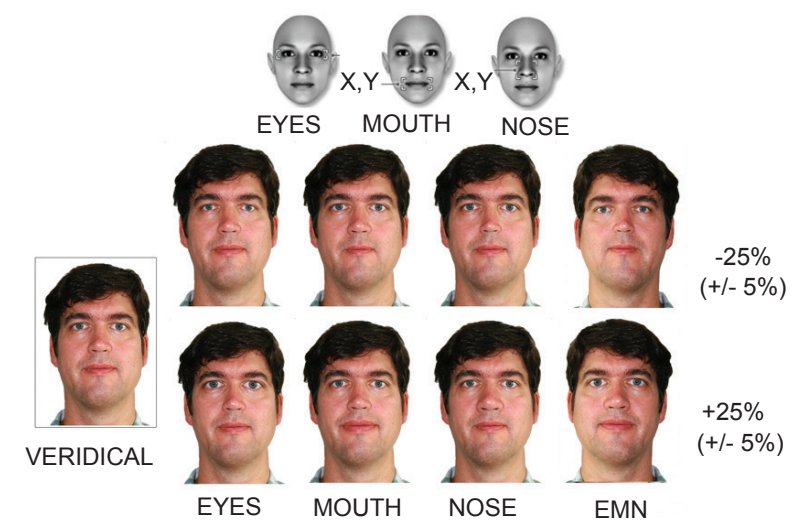

Figure 1. Schematic representation of possible size changes in facial features by $25 \%( \pm 5 \%)$ in relation to the veridical image. Top: schematic representation of the changes made to the eye, nose and mouth. Middle row: examples of decrements in the size of the eyes, the mouth, the nose, and EMN (simultaneous changes to eyes, mouth and nose) (in mirror orientation). Bottom: examples of increments in size. The veridical image is shown on the far left. 


\section{Results}

\section{Self-face recognition}

Accuracy. In this two-alternative forced choice task, the participants had to choose which of the two self-faces (veridical vs. clone) presented on the screen was their veridical face. One participant (female) was excluded from the final analysis due to outlier values.

The accuracy with respect to veridical self-faces varied with facial feature $(F(2.20,68.33)=19.34, p<$ $\left..0001, p \eta^{2}=.38\right)$ and with feature size $(F(1,31)=12.41$, $\left.p=.0001, p \eta^{2}=.29\right)$. The participants identified selffaces with their veridical eyes with a mean accuracy of $88 \%(S E=3)$. However, the accuracy for their veridical mouth $($ Mean $=75 \%, S E=4)$ and nose (Mean $=76 \%, S E$ $=3$ ) was lower (Figure 2a). Self-face recognition with multiple feature distortions (EMN; Mean $>95 \%$ ) was easier than with local, individual distortions $(p<.001)$. There was also an interaction between features and size $\left(F(2.40,74.50)=5.06, p=.006, p \eta^{2}=.14\right)$ : accuracy to EMN and eyes was independent of feature size, whereas the accuracy to nose and mouth was higher when selffaces were paired with larger clones (+clones) than smaller ones (-clones) $(p=.001)$.

Reaction time. Reaction time varied significantly with facial feature $(F(2.44,75.76)=11.23, p<.0001$, $\left.p \eta^{2}=.27\right)$ but not with feature size $(F(1,30)=0.11, p$ $=.74$ ). The participants were significantly faster (and more accurate) at self-recognition against clones with multiple and simultaneous rather than local feature distortions $(p=.002)$ (Figure 2b).

\section{Self-face preferences}

In this task, the participants had to choose which self-face image they preferred: the self-face or the clone. The participants (one outlier) tended to choose their veridical images over their clones $(F(2,66)=$ $\left.6.34, p=.003, p \eta^{2}=.16\right)$. The preference for cloned features varied with size $\left(F(1,33)=5.04, p=.03, p \eta^{2}\right.$ $=.13$ ) and a reliable interaction between facial features and size was observed $\left(F(2,66)=6.84, p=.002, p \eta^{2}=\right.$ .17). The participants preferred smaller noses to larger noses $(t(33)=-3.40, p=.002$; Figure $2 \mathrm{c})$, even though many participants did not notice when their nose size was decreased by the researchers in the first part of the experiment. About half of the participants preferred their nose to be half of its original area (at least when in frontal view). The participants did not show a clear preference for clones or veridical faces when features were increased or enlarged simultaneously (EMN, 3-5\% range).

\section{Experiment 2}

Experiment 1 indicated that the participants had a distorted perception of the size of their facial features. This experiment aimed to create a more detailed snapshot of the mental representation of one's own face using a wider range of feature distortions.
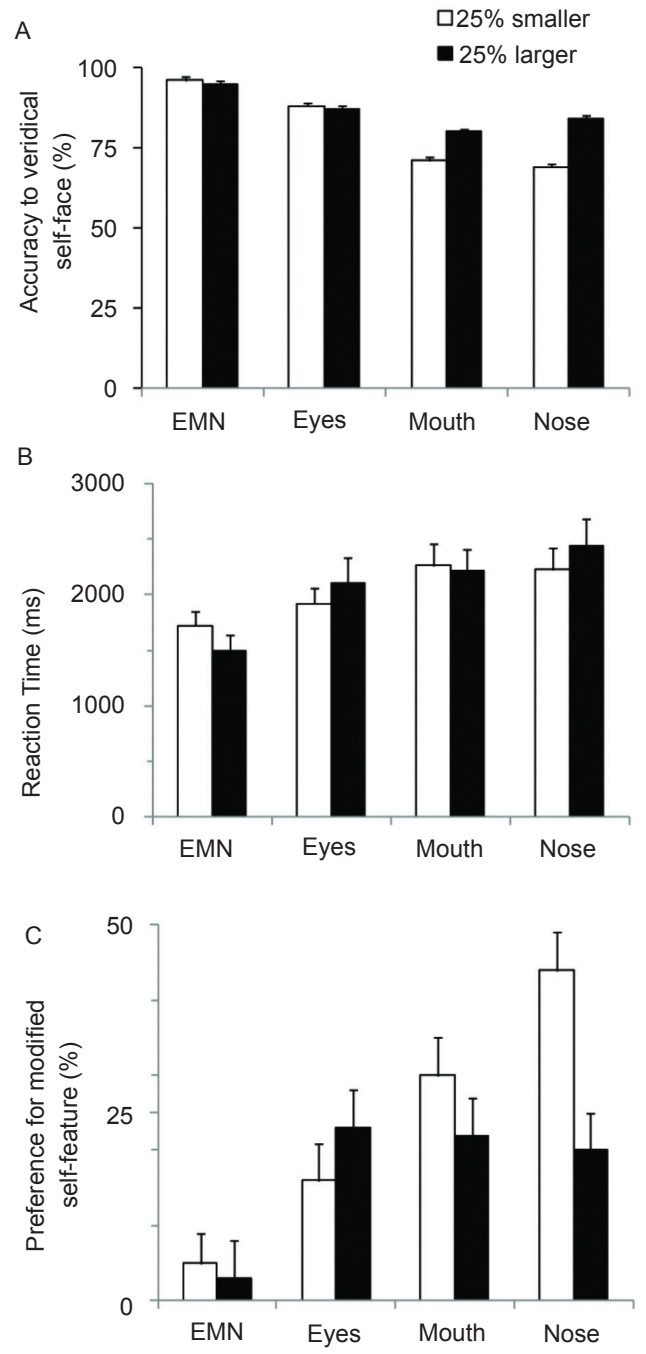

Figure 2. Accuracy for veridical self-faces (A) and reaction time for their accurate recognition (B). Preference for manipulated facial features (C). Size of facial features increased or decreased in the area by $25 \%( \pm 5)$. EMN: simultaneous changes to eyes, mouth and nose $( \pm \mathrm{SE})$.

\section{Method}

\section{Participants and method}

Undergraduate students and staff members were recruited using internal mail $\left(N=33 ; 24\right.$ females; $M_{\text {age }}$ $=33$ years; $S D=11$ ).

The stimuli were acquired and processed as in Experiment 1 except for the range of feature distortions used. The area of the facial features was increased or decreased in size in 10-integer steps, which created 10 face clones after each transformation (5 clones with features larger and 5 clones with features smaller than the features in the veridical image). Three size ranges were used to circumvent individual differences in recognition threshold (range $1= \pm 50 \%$; range $2=$ $\pm 32 \%$; range $3= \pm 20 \%$ ). Mirror versions were created by flipping images horizontally. 


\section{Procedure}

Each participant saw 640 images in total: 4 facial features $\mathrm{x} 2$ orientations (normal vs. mirrored) $\mathrm{2}$ visual fields $\times 10$ size manipulations, with each cycle repeated 4 times. Hence, participants saw their veridical image 320 times in the normal orientation and 320 times in the mirror orientation and 8 times each of the 80 manipulated features. The veridical images were repeatedly presented and it was expected that participants would add the photograph to the internal representation of their own face (Carbon \& Leder, 2005), which could counterbalance some of the difficulties in self-face recognition with 2D representations of faces and are also subject to some technical constraints such as camera model, lens, focal length and illumination (among others).

\section{Results}

Data points were fit (maximum likelihood) with the standard normal distribution:

$$
f(x)=\frac{1}{\sqrt{2 \pi \sigma^{2}}} e^{-\frac{(x-\mu)^{2}}{2 \sigma^{2}}}
$$

where $\mathbf{x}$ refers to the size changes of a given facial feature. Linear regression fits $(r>85 \%)$ resulted in similar thresholds when participants' responses reached $75 \%$ accuracy.

Figure 3 shows the recognition thresholds for the female participants only, since the number of males was not large enough to allow comparisons. The only clear gender difference observed was related to the thresholds for mouth recognition: male thresholds when paired with -clones $($ Mean $=27 \%)$ were higher than female thresholds (Mean $=13 \%)$. However, the gender difference was smaller when self-faces were paired with + clones $\left(\right.$ Mean male $=17 \%$, Mean $\mathrm{f}_{\text {emale }}=11 \%$ ). Large individual differences in the recognition of self-features were observed across all size ranges. To circumvent this problem, thresholds for each size distortion of a facial feature were averaged across male and female participants for each of the three size ranges presented.

Self-face recognition was easier when veridical faces were paired with +clones than-clones. EMN accuracy was high, which led to overall low thresholds. Nonetheless, EMN thresholds with + clones $($ Mean $=6 \%)$ were lower than with -clones (Mean $=9 \%)$. The estimation of nose size $\left(M_{\text {nose }}=28 \%, M_{\text {nose }}=23 \%\right)$ was harder than the eye size $\left(M_{\text {eye- }}=11 \%, M_{\text {eyet }}=8 \%\right)$ and mouth size $M_{\text {mouth }-}=$ $22 \%, M_{\text {mouth }+}^{\text {eye- }}=13 \%$ ) estimation in all pairings.

The averaged thresholds for mirrored self-features were slightly higher than those obtained with the normal orientation, but not for all participants and not consistently across features. In addition, no clear differences were observed between males and females with the facial features analysed, but a further study with a larger number of male participants is needed to confirm that result.

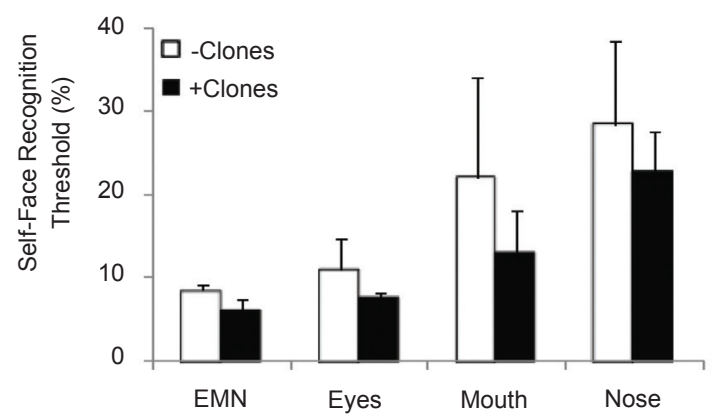

B

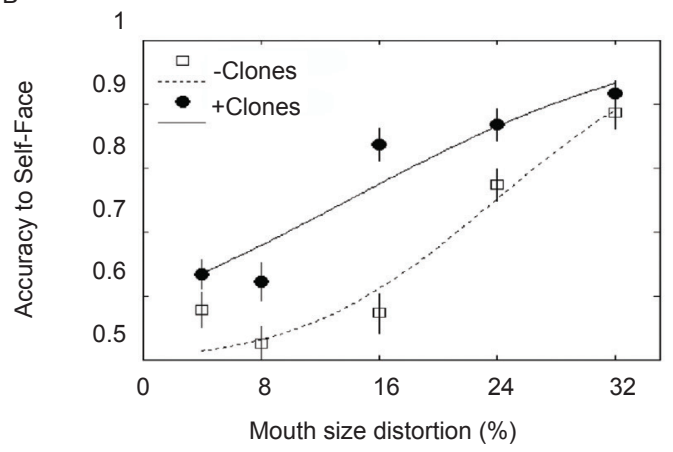

Figure 3. (A) Self-face recognition thresholds (females only). (B) Example of accuracy to one's mouth in pairings with mouth clones. Pairings of self-faces with +clones (filled circles) and -clones (open squares). The solid and dotted curves are a maximum-likelihood fit of the standard normal distribution to one of the datasets $( \pm \mathrm{SE})$.

\section{Experiment 3}

As mentioned in the introduction, when we observe our own faces (e.g., in a mirror) we virtually assume the perspective of other people towards ourselves. Accordingly, one could say that to investigate self-face perception is to investigate the self as a social object. Therefore, this experiment investigated if and how participants would change their facial features to conform to implicit notions of facial attractiveness (either from their own standpoint or from the standpoint of another). The experiment further investigated whether and to what extent such facial manipulations would be linked to self-esteem. Our hypothesis was that the lower the selfesteem, the larger the facial manipulations would be.

\section{Method}

\section{Participants}

Undergraduate psychology students $(N=41 ; 37$ females, Mean age $=23$ years, $S D=3$ ) were recruited by email. The 4 males were excluded from the analysis due to their small number.

\section{Procedure}

On the day the photographs were taken, the participants were asked to complete the SSES questionnaire. About 10 days later participants had a practice session prior to testing and when they were confident they could use the software 
for face manipulation, the digital photograph of their face was uploaded. The two tasks to be completed were presented in a randomised and counterbalanced order.

The instruction for the 'attractive to self' task was as follows: "In this task, you can manipulate the image of your face to make it more attractive to yourself. You do not need to change your facial features if you are happy with them. If you manipulate the image but you are not happy with the final result, remember that you can return to your veridical image". The instruction for the 'attractive to others' task was as follows: "In this task, you can manipulate the image of your face to make it more attractive to other people. [...then as above]". A repeated-measures, within-subject design was used.

The average of the increase or decrease in feature size in the $\mathrm{x}$ and $\mathrm{y}$ axis (in absolute values) was used to calculate the magnitude of facial size changes for each self-feature.

\section{Results}

A repeated measures 3 (facial features: eyes, nose, mouth) $\times 2$ (magnitude of size increases and decreases) ANOVA revealed a significant difference in the magnitude of change between features $(F(1.57$, $56.62)=4.84, p=.01, p \eta^{2}=.12$ ), but there were no significant differences between the 'attractive to self' and 'attractive to others' conditions $(F<1)$. Pairwise comparisons showed that the magnitude of mouth changes differed significantly from the eye changes $(p=.04)$ (Figure 4a). It is important to note that the mouth changes to increase self-attractiveness were bidirectional: some participants increased the size of their mouth, while others decreased it.

Next, we investigated whether there was a correlation between the magnitude of facial changes and SSES scores. We found that participants with high SSES scores tended to make smaller changes to their features than the participants with lower scores. Significant correlations between SSES scores and changes in the 'attractive to self' condition were found for the eyes $(r=$ $-.45, p=.006)$, the mouth $(r=-.33, p=.05)$ and the nose $(r=-.40, p=.01)$. Nose changes were also significantly correlated to SSES scores in the 'attractive to others' condition ( $r=-.43, p=.008)$ (Figure 4).

In the 'attractive to self' condition, the SSES performance factor was significantly correlated with mouth changes $(r=-.37, p=.03)$, the SSES social factor was correlated with eyes $(r=-.36, p=.03)$ and nose changes $(r=-.35, p=.03)$, and the SSES appearance factor was associated with eyes $(r=-.42, p=.01)$ and nose changes $(r=-.35, p=.04)$. In the 'attractive to others' condition, the SSES social and appearance factors were correlated with nose changes $(r=-.44, p=.007$ and $r=-.33, p=.04$, respectively). Multiple regression analysis showed that SSES scores in the 'attractive to self' condition explained $20 \%$ of the variance in eye size $(F(1,35)=8.72, p=.006), 11 \%$ of the variance in mouth size $(F(1,35)=4.29, p=.05)$, and $16 \%$ of the variance in nose size $(F(1,35)=6.68, p=.01)$. The SSES scores in the 'attractive to others' condition explained of the $18 \%$ variance in nose size $(F(1,35)=7.81, p=.008)$.
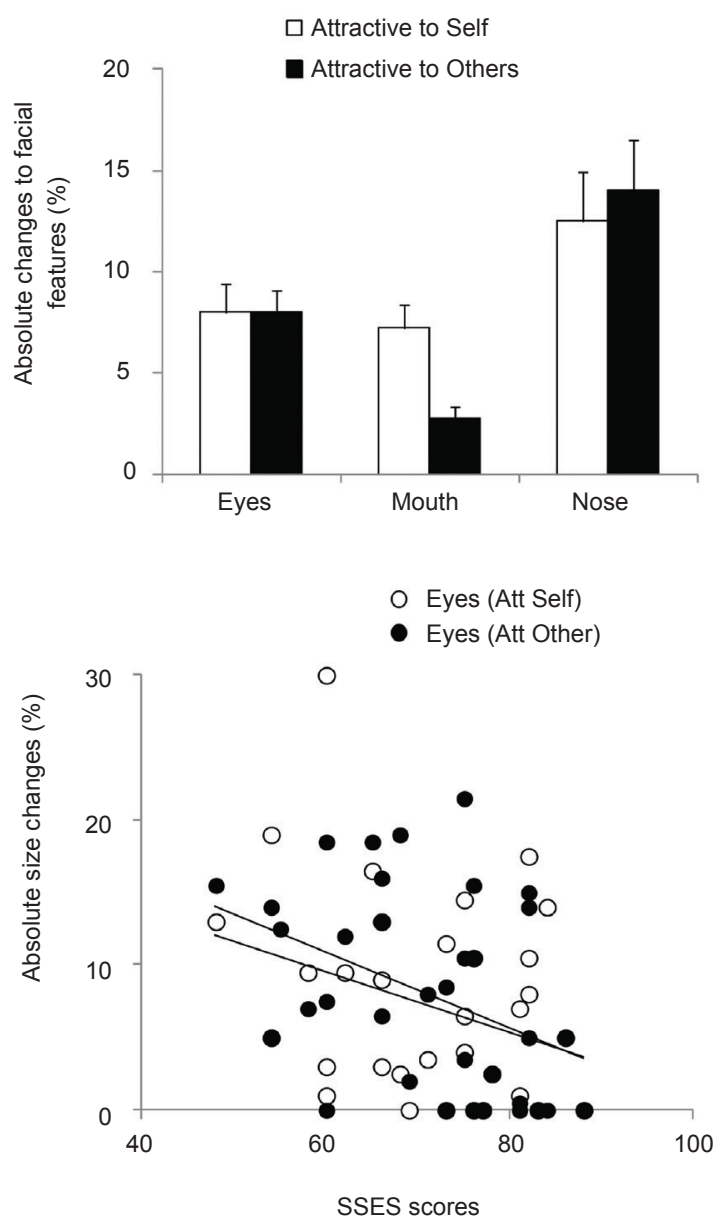

Figure 4. Changes to facial features to make self-faces more attractive to oneself ('Attractive to Self') or more attractive to what participants believed other people would find attractive ('Attractive to Other') and the association of such changes with state self-esteem. (Top) Average changes to facial features in the two conditions ( $\pm \mathrm{SE}$ ). (Bottom) Relationship between the magnitude of eye size changes and State Self-Esteem Scale (SESS) scores.

\section{Discussion}

This study examined different aspects of selfface perception and possible links between selfface recognition, self-esteem and internal notions of attractiveness. It combined novel tasks designed to measure the accuracy of facial self-representation and the perception of self-face attractiveness with a measure of state self-esteem.

Experiment 1 revealed that it was easier to recognise one's veridical face when it was paired with clones with larger rather than smaller features. Overall accuracy varied with the facial feature tested: one's veridical eye size was easier to recognise than one's 
mouth size, whereas nose size had the lowest accuracy. About $1 / 4$ of the participants preferred clones with larger eyes and almost $1 / 2$ of them preferred clones with smaller noses. This finding is in line with reports that rhinoplasty and blepharoplasty were some of the most common cosmetic procedures (Alsarraf, Larrabee Jr., \& Johnson Jr., 2001) and there is evidence that improved facial appearance affects the self-esteem and the mood of patients (Nicodemo, Pereira, \& Ferreira, 2008).

The preference for smaller noses and larger eyes, even in participants with average or high SSES scores, seems to indicate a general preference for neotenous facial features that could be explained in the context of the Sexual Selection Theory. Neotenous features have been linked to youth, fertility, and good health and therefore are highly valued when choosing a mating partner (Barber, 1995; Honeköpp, Bartholomé, \& Jansen, 2004).

Of particular note is that the preference bias towards neotenous features reported here concerns the participants' preferences for neotenous features in themselves (rather than in others). Because the self only develops in relation to others, one would expect that features that are rated as attractive by the social environment become internalised and form part of one's ideal self-image. This relationship seems particularly salient with respect to self-face perception because when we see our own faces we are virtually assuming the perspective of the other towards ourselves. Accordingly, one might say that to investigate self-face perception is to investigate the self as a social object. In line with the thought that subjects are particularly (self-)aware of features that contribute to a neotenous appearance, self-face recognition thresholds (Experiment 2) suggest that most participants underestimated the actual size of their eyes and mouths but overestimated the size of their noses. There was an advantage of multiple and simultaneous size changes over single feature changes, in line with accounts of good recognition when changes were made simultaneously to eyes, mouth and nose (Farah, Wilson, Drain, \& Tanaka, 1998).

Although the size of one's facial features is not the sole contributor to physical attractiveness, when provided with the opportunity, the majority of the participants in Experiment 3 manipulated the size of their facial features. As predicted by the SelfDiscrepancy Theory, there were significant inverse correlations between state self-esteem and changes to eyes, mouths and noses when participants aimed at increasing attractiveness of their faces to themselves; in other words, the lower the self-esteem, the larger the size of the changes. Interestingly, when attempting to increase their facial attractiveness to others, many participants with lower SSES scores manipulated their noses, whereas no significant correlation with state self-esteem was found with the magnitude of eye and mouth manipulations. This raises potentially interesting questions for further research into how social factors modulate notions of self-face attractiveness (Diener, Wolsic, \& Fujita, 1995).

Overall, the results suggest that one's body image (in this case, the representation of one's face) can be highly influenced by social factors in the sense that features that are generally assumed to be attractive in others (and that we believe others find attractive) are also desired by ourselves. In other words, if neotenous facial features are what we find attractive in others (and what we know others find attractive) - in line with predictions based on the Sexual Selection Theory - and if one's self-image develops in relation to others, then one would expect a bias towards neotenous features with regard to one's ideal self. This expectation was at least partly confirmed by the present study. In addition, the extent to which people are inclined to manipulate their facial features when provided with the opportunity is correlated with lower self-esteem.

In summary, based on Higgins' Self-Discrepancy Theory and the Sexual Selection Theory, we predicted that discrepancies between internal notions of selfattractiveness (i.e., discrepancies between internal representations of one's actual face and one's ideal face) would correlate with lower self-esteem and that participants would display a preference toward neotenous facial features. Both predictions were partly confirmed. In addition, this study provides new insights into the accuracy of facial self-representation and raises interesting questions for future research. The results contribute to the understanding of some aspects of selfhood, since the reported perceptual biases could reinforce the distinctiveness of one's face. Although the internal representation of one's facial features varied considerably among participants, the perceptual biases indicate a general tendency to underestimate one's eye size but to overestimate the size of one's nose. However, a certain tolerance to error might be intrinsic to the internal representation of self-faces to accommodate a facial identity that remains consistent during one's life span.

\section{Acknowledgements}

We would like to thank research students Kristina Gorodeski, Suet Man Chan, Yuki Sato, and Marilena Schembri for their help with data collection.

\section{Conflict of interest statement}

On behalf of all authors, the corresponding author states that there is no conflict of interest.

\section{References}

Alsarraf, R., Larrabee Jr., W. F., \& Johnson Jr., C. (2001). Cost outcomes of facial plastic surgery. Archives of Facial Plastic Surgery, 3, 44-47.

Barber, N. (1995). The evolutionary psychology of physical attractiveness: sexual selection and human morphology. Ethology and Sociobiology, 16, 395-424. 
Blais, C., Jack, R. E., Scheepers, C., Fiset, D., \& Caldara, R. (2008). Culture shapes how we look at faces. PLoS ONE, 3, e3022.

Brédart, S., \& Young, A. W. (2004). Self-recognition in everyday life. Cognitive Neuropsychiatry, 9, 183-197.

Bruce, V., \& Young, A. (1986). Understanding face recognition. British Journal of Psychology, 77, 305-327.

Carbon, C. C., \& Leder, H. (2005). Face adaptation: changing stable representations of familiar faces within minutes? Advanced Experimental Psychology, 1, 1-7.

DeBruine, L. M., Jones, B. C., Tybur, J. M., Lieberman, D., \& Griskevicius, V. (2010). Women's preferences for masculinity in male faces are predicted by pathogen disgust, but not by moral or sexual disgust. Evolution and Human Behavior, 31 69-74.

Devue, C., \& Brédart, S. (2011). The neural correlates of visual selfrecognition. Consciousness and Cognition, 20, 40-51.

Devue, C., Collette, F., Balteau, B., Degueldre, C., Luxen, A., Maquet, P., \& Brédart, S. (2007). Here I am: the cortical correlates of visual self-recognition. Brain Research, 1143, 169-182.

Diener, E., Wolsic, B., \& Fujita, F. (1995). Physical attractiveness and subjective well-being. Journal of Personality and Social Psychology, 69, 120-129.

Farah, M. J., Wilson, K. D., Drain, M., \& Tanaka, J. (1998). What is "special" about face perception? Psychological Review, 105, 482-498.

Gallagher, S. (1986). Body image and body schema: a conceptual clarification. Journal of Mind and Behavior, 7, 541-554.

Gallagher, S. (2012). The body in social context: some qualifications on the 'warmth and intimacy' of bodily self-consciousness. In K. Crone, K. Musholt \& A. Strasser (Eds.), Facets of self-consciousness (Vol. 84, pp. 91-121): Grazer Philosophische Studien.

Gallup, G. G. (1970). Chimpanzees: self-recognition. Science, 167, 86- 87.

Gallup, G. G. (1998). Self-awareness and the evolution of social intelligence. Behavioural Processes, 42, 239-247.

Garrido, L., Duchaine, B., \& Nakayama, K. (2008). Face detection in normal and prosopagnosic individuals. Journal of Neuropsychology, $2,119-140$

Heatherton, T. F., \& Polivy, J. (1991). Development and validation of a scale for measuring state self-esteem. Journal of Personality and Social Psychology, 60, 895-910.

Higgins, E. T. (1987). Self-discrepancy: a theory relating self and affect. Psychological Review, 94, 319-340.

Higgins, E. T. (1989). Self-discrepancy theory: what patterns of selfbeliefs cause people to suffer? Advances in Experimental Social Psychology, 93-136.

Hodzic, A., Muckli, L., Singer, W., \& Stirn, A. (2009). Cortical responses to self and others. Human Brain Mapping, 30, 951-962.
Honeköpp, J., Bartholomé, T., \& Jansen, G. (2004). Facial attractiveness, symmetry, and physical fitness in young women. Human Nature, 15, 147-167.

Kandrik, M., \& DeBruine, L. M. (2012). Self-rated attractiveness predicts preferences for opposite-sex faces, while self-rated sextypicality predicts preferences for same-sex faces. Journal of Evolutionary Psychology, 10, 177-186.

Keyes, H., \& Brady, N. (2010). Self-face recognition is characterized by "bilateral gain" and by faster, more accurate performance which persists when faces are inverted. The Quarterly Journal of Experimental Psychology, 63, 840-847.

Kircher, T. T. J., Senior, C., Phillips, M. L., Rabe-Hesketh, S., Benson, P. J., Bullmore, E. T. ... \& David, A. S. (2001). Recognizing one's own face. Cognition, 78, B1-B15.

Lewis, M. (2003). The emergence of consciousness and its role in human development. Annals of the New York Academy of Sciences, 1001, 104-133.

Ma, Y., \& Han, S. (2009). Self-face advantage is modulated by social threat: boss effect on self-face recognition. Journal of Experimental Social Psychology, 45, 1048-1051.

Mead, G. H. (1962). Mind, Self and Society. Chicago: The University of Chicago Press.

Nicodemo, D., Pereira, M. D., \& Ferreira, L. M. (2008). Self-esteem and depression in patients presenting Angle Class III malocclusion submitted for orthognathic surgery Medicina Oral Patologia Oral y Cirurgia Bucal, 13, E48-E51.

Platek, S. M., Keenan, J. P., Gallup, G. G. J., \& Mohamed, F. B. (2004). Where am I?: the neurological correlates of self and other. Cognitive Brain Research, 19, 114-122.

Rooney, B., Keyes, H., \& Brady, N. (2012). Shared or separate mechanisms for self-face and other-face processing?: evidence from adaptation. Frontiers in Psychology, 3, 1-9.

Russel, R., Duchaine, B., \& Nakayama, K. (2009). Super-recognizers: People with extraordinary face recognition ability. Psychonomic Bulletin and Review, 16, 252-257.

Suddendorf, T., Simcock, G., \& Nielsen, M. (2007). Visual selfrecognition in mirrors and live videos: evidence for a developmental asynchrony. Cognitive Development, 22, 185-196.

Sugiura, M., Sassa, Y., Jeong, H., Wakusawa, K., Horie, K., Sato, S., \& Kawashima, R. (2012). Self-face recognition in social context. Human Brain Mapping, 33, 1364-1374.

Uddin, L. Q., Kaplan, J. T., Molnar-Szakacs, I., Zaidel, E., \& Iacoboni, M. (2005). Self-face recognition activates a frontoparietal "mirror" network in the right hemisphere: an event-related fMRI study. NeuroImage, 25, 926-935.

Zahavi, D., \& Roepstorff, A. (2011). Faces and ascriptions: Mapping measures of the self. Consciousness and Cognition, 20, 141-148. 